\title{
VIER WICHTIGE VERFASSUNGSRECHTLICHE ENTSCHEIDUNGEN DES JAPANISCHEN OBERSTEN GERICHTS
}

\author{
Von Georg Tagami
}

\section{Wahlgesetz}

In dem Verfahren über die Nichtigerklärung der Wahl der Oberhausmitglieder hat das Oberste Gericht durch Plenarentscheidung vom 5. Februar 1964 den Antrag, die Wahl für nichtig zu erklären, weil die Sondertabelle ${ }^{2}$ zum Wahlgesetz vom 15. April 1950 über die Zahl der Abgeordneten im Verhältnis zur Zahl der Wahlberechtigten in jedem Wahlkreis nicht revidiert worden sei, zurückgewiesen, weil das Gesetz den Gleichheitsgrundsatz nicht verletzt und deshalb nicht gegen Art. 14 Abs. 1 der Verfassung verstößt. Die japanische Verfassung schreibt vor, daß die Zahl der Mitglieder jedes Hauses durch Gesetz bestimmt wird und daß die Wahlkreise, das Wahlverfahren und weitere mit der Wahl der Parlamentsmitglieder zusammenhängende Fragen durch Gesetz geregelt werden. Daher kann man sagen, daß es grundsätzlich dem Ermessen des Parlaments überlassen ist, diese Angelegenheiten zu regeln. Darum ist das Parlament befugt, vorzuschreiben, nach welchem Verhältnis die Zahl der im Wahlkreis gewählten Abgeordneten dem einzelnen

1 Hier habe ich vier wichtige Urteile des japanischen Obersten Gerichts ausgewählt. Das erste Urteil bezieht sich auf eine politische Frage, die gerichtlich nicht zu lösen und demzufolge nicht überprüfbar zu sein scheint. Das zweite hat mit der Nachprüfbarkeit von Ermessensermächtigungen zu Eingriffen in die Freiheit des einzelnen zu tun. Das dritte geht sowohl das Verhältnis vom Beamtenrecht zum Arbeitsrecht als auch das Wesen des Gemeinwohls als Schranken der Grundrechtsgarantie an. Schließlich betrifft das vierte nicht nur die leistende Verwaltung, sondern auch das Problem einer Programmvorschrift unserer Verfassung.

2 Während die erste Sondertabelle zum Wahlgesetz sich auf die Wahlkreise des Unterhauses und die jedem Wahlkreis zugeteilten Abgeordnetenzahl bezieht, so betrifft die zweite Sondertabelle diejenigen des Oberhauses. Unser Oberhaus besteht aus 250 Abgeordneten, von denen 100 aus dem ganzen Lande und die übrigen 150 in Wahlkreisen gewählt werden. Alle Abgeordneten, sowohl des Oberhauses als auch des Unterhauses sind Vertreter des ganzen Volks. Japan hat seit der alten Verfassung im, ahre 1889 das Zweikammersystem aufgenommen. Die wichtigen Wahlkreises und ihre Zahl der Oberhausmitglieder und der betreffenden Bevölkerung sind folgende:

\begin{tabular}{|c|c|c|c|}
\hline \multirow[t]{2}{*}{ (Wahlkreis) } & \multirow[t]{2}{*}{ (Zahl der Mitglieder) } & \multicolumn{2}{|c|}{ (Bevölkerungszahl) } \\
\hline & & 1946 & 1969 \\
\hline Residenzstadt Tokio & 8 & 4 (Millionen) & 11 \\
\hline Provinz Hokkaido & 8 & 3,5 & 5,4 \\
\hline Präfektur Osaka & 6 & 3 & 7 \\
\hline Präfektur Aizi & 6 & 3 & 5,2 \\
\hline Präfektur Hyogo & 6 & 2,8 & 4,6 \\
\hline Präfektur Kanagawa & 4 & 2 & 5,2 \\
\hline Präfektur Gumma & 4 & 1,5 & 1,7 \\
\hline Präfektur Okayama & 4 & 1,5 & 1,7 \\
\hline Präfektur Tozigi & 4 & 1,5 & 1,6 \\
\hline Präfektur Tottori & 2 & 0,5 & 0,6 \\
\hline
\end{tabular}

Im Jahre 1946 hatte man die Zahl der Abgeordneten im Verhältnis zur Bevölkerung jedem Wahlkreis zugeteilt. Durch die Konzentration der Bevölkerung auf Großstädte hat sich dennoch die Sachlage geändert. In der Residenzstadt Tokio kommt ein Abgeordneter auf 1400000 Wähler, während in der Präfektur Tottori ein Abgeordneter auf 293400 Wähler entfällt. Eben dieses Mißverhältnis war der Grund für die Anfechtung der zweiten Sondertabelle wegen Verfassungswidrigkeit. Unser Regierungsausschuß zur Reform des Wahlsystems hat im letzten Mai ein Gutachten über die Anderung der zweiten Sondertabelle erstattet, obgleich das betreffende Anderungsgesetz noch heute nicht beschlossen wurde. Demzufolge soll die Abgeordnetenzahl der Präfekturen Osaka und Kanagawa um zwei erhöht und zugleich diejenige der Präfekturen Tozigi, Gumma und Okajama um zwei vermindert werden. 
Wahlkreis zuteil werden soll. Unter den Vorschriften der Verfassung, vor allem Art. 14 und 44, gibt es keine Bestimmung, nach der die Zahl der Abgeordneten in jedem Wahlkreise im Verhältnis zu der entsprechenden Wählerzahl festgesetzt werden muß. Vom Verfassungsgrundsatz der Rechtsgleichheit aus ist es zwar zweckmäßig, die Zahl der Abgeordneten in dieser Weise festzusetzen. Jedoch kann man bei dieser Zuteilung einige andere Maßstäbe berücksichtigen, wenn auch die Einwohnerzahl des Wahlkreises dabei die Hauptrolle spielen wird. Die Wahl der Hälfte der Mitglieder des Oberhauses findet alle drei Jahre statt. Daraus ergibt sich, daß es schwer ist, die Zahl der Abgeordneten eines Walkreises ungerade zu bestimmen. Außerdem muß man die Größe, die geschichtliche Entwicklung jedes Wahlkreises, die Zuteilung mindestens eines Abgeordneten zu jedem Verwaltungsbezirk usw. berücksichtigen, was keineswegs unvernünftig ist. Solange dem Parlament im Hinblick auf die Entscheidung über diese Verteilung Ermessensspielraum eingeräumt ist, kann man nicht das verschiedene Verhältnis zwischen der Zahl der Abgeordneten und der Wählerzahl in jedem Wahlkreis schlechthin als Verstoß gegen Art. 14. Abs. 1 der Verfassung betrachten und damit für nichtig erklären, es sei denn, daß die Verteilung der Abgeordneten des Wahlkreises eine äußerste Ungleichheit zur Folge haben würde. Daß die Sondertabelle zum geltenden Wahlgesetz, was das Verhältnis der Wählerzahl zur Zahl der Abgeordneten anlangt, noch nicht revidiert worden ist, zieht zwar notwendig Ungleichheiten nach sich. Sofern der oben erwähnte Grad nicht überschritten wird, handelt es sich trotzdem um die Richtigkeit der Gesetzgebungspolitik, ohne daß die Verfassungsmäßigkeit dieser Regelung in Frage gestellt wird. Aus diesem Grunde ist der Antrag zurückgewiesen worden.

Richter Saito hat sich gegen diese Mehrheitsansicht ausgesprochen. Er bezweifelt, daß diese Ansicht auch in dem nur ausnahmsweise angenommenen Fall, daß man die Sondertabelle zum Wahlgesetz von Verfassungs wegen für nichtig erklären könne, richtig sei, weil die Plenarentscheidung des Obersten Gerichts vom 16. Dezember 1958 (Rechtsfall Sunagawa) den Sicherheitsvertrag als außer dem Bereich des richterlichen Prüfungsrechts erklärt habe, es sei denn, daß der Vertrag auf den ersten Blick für verfassungswidrig und damit nichtig angesehen werde. Natürlich sei es der Ausdruck des Eifers, die Autorität der Justiz zu verteidigen, wenn man solch einen Ausnahmefall annehme, auf den sich die richterliche Prüfung erstrekken könne, während man denselben Fall regelmäßig aus dem Bereich der Justiziabilität ausschließe. Diese geistige Haltung eines Richters habe wohl den Zweck, der Verteidigung der Grundrechte der Staatsbürger zu dienen. Es sei aber zweifelhaft, ob man dadurch wirklich die Autorität der Justiz erhöhen und das Vertrauen des Volks gewinnen könne. Er habe die Minderheitsansicht des Richters Frankfurter in dem Urteil des Obersten Gerichts der Vereinigten Staaten in dem Prozeß Baker v. Carr vom 26. März 1962 durchgelesen und dabei empfunden, daß man die oben erwähnten Probleme noch einmal bedenken sollte. Frankfurters Ansicht lautet: „Die Autorität eines Gerichts, welches weder Reichtum noch Waffen hat, hängt zuletzt von der moralischen Unterstützung des Volkes ab. Um solch eine Volksmeinung zu pflegen, soll sich das Gericht sowohl wirklich als auch dem Schein nach aus dem politischen Streit heraushalten, anders ausgedrückt, sich nicht in den Konflikt der politischen Mächte um die politische Entscheidung verwickeln. Die Aufrechterhaltung der einer wirksamen Ausübung der Justiz innewohnenden Schranken ist viel wichtiger als die Erweiterung der Sphäre der Justiziabilität.“ Es gibt noch viele andere Elemente als das Verhältnis zur Wählerzahl, um die Zahl der 
Abgeordneten eines jeden Wahlkreises zu bestimmen. Es ist bemerkenswert, daß man „keine ausreichende Richtschnur der richterlichen Beurteilung finden kann“, um über diese Elemente zusammenfassend $\mathrm{zu}$ urteilen. Wörtlich sagt Richter Saito: „Dem Richter in dieser Hinsicht die Entscheidungszuständigkeit zu gewähren, heißt nichts anderes als ihn mit der Autorität Gottes auszustatten. Unter unserer Verfassung wird nicht Rechtsschutz gegen jeden politischen Fehler und jede ungerechte Ausübung der Gesetzgebungsgewalt gewährleistet.“ Es ist nicht festzustellen, bei welcher Sachlage der Ausnahmefall nach der Mehrheitsansicht vorliegen soll. Vielleicht kommen auch in Zukunft kaum Umstände vor, die dem erwähnten Ausnahmefall entsprechen und die Wahl nichtig machen. Es ist viel wichtiger, sich auf den Druck der öffentlichen Meinung und auf die Vernunft des Gesetzgebungs- und Verwaltungsorgans zu verlassen. Jede Klage, welche wegen eines solchen Ausnahmefalls erhoben würde, müßte bei der Unklarheit des Tatbestandes eines Ausnahmefalles zurückgewiesen werden. Demzufolge würde ein solcher Prozeß nicht nur wider die Erwartung des Volks verlaufen, sondern auch Mißtrauen in die Justiz nach sich ziehen. Wenn die Sondertabelle zum Wahlgesetz gegen den Gleichheitssatz der Verfassung verstoßen und die Nichtigkeit der Wahl herbeiführen würde, würde das letzten Endes bedeuten, daß dem Volk das gegenwärtige Gesetzgebungsorgan genommen würde, ohne daß dieses ein Ersatzorgan zu wählen imstande wäre. Solch eine Sachlage könnte zur Vernichtung des Staatsorgans führen, denn die Nichtigkeit der Wahl der Hälfte der Oberhausmitglieder unterbricht die Funktion des Parlaments überhaupt. Der Prozeß nach $\ 204$ Wahlgesetz zielt eigentlich darauf ab, Fehler in bezug auf die Organisation und Durchführung einer Wahl zu berichtigen und erforderlichenfalls die Wahl für nichtig zu erklären. Die Wiederwahl, die jener Prozeß zur Folge hat, soll innerhalb von $40 \mathrm{Ta}-$ gen nach dem Tag stattfinden, an welchem der Grund zur Wiederwahl sich ereignet. Wenn in diesem Fall die Sondertabelle zum Wahlgesetz für nichtig erklärt würde, stellte sich die Frage, ob man die Tabelle innerhalb dieser kurzen Frist revidieren kann. Wenn nicht, kann man nichts anderes tun, als die nichtige Wahl zu wiederholen. Ein Versuch, die Fragen, hinsichtlich derer das Parlament zu der Regierung in erheblichem $\mathrm{Maß}$ in Widerspruch stehen mag, gerichtlich $\mathrm{zu}$ lösen, wird eine nicht in Ordnung zu bringende Verwirrung hervorbringen. In diesem Sinne zweifle er, so sagt Richter Saito, besonders daran, ob im Wahlprozeß nach $₫ 204$ Wahlgesetz der Anspruch auf richterliche Prüfung der Verfassungsmäßigkeit ipso iure geltend gemacht werden kann. In Japan kennt man nur die Mehrheitswahl im Wahlkreis. Hinsichtlich der Verhältniswahl gilt dagegen weder die Vollproporz, noch das englische, einfache übertragbare Abstimmungssystem. Darum läuft die aus der Gleichheit vor dem Gesetz gefolgerte Gleichheit der Wahl im Grunde genommen mit der Allgemeinheit der Wahl auf eines hinaus in dem Sinne, daß einerseits das System der nochmaligen Abstimmung eines bestimmten Teils der Wähler verboten und damit der Zählwert einer Stimme gleichgemacht wird, andererseits die Zahl der Wahlberechtigten sich der Gesamtzahl der politisch urteilsfähigen Staatsbürger möglichst annähert. Wenn man auch die Zahl der Abgeordneten im Verhältnis zur Wählerzahl jedes Wahlkreises festlegen kann, so sind dennoch die gewonnenen Stimmen jedes Gewählten infolge unübertragbarer Stimmabgabe regelmäßig voneinander und sogar die Gesamtzahl der gültigen Stimmen wegen Freiheit der Stimmabgabe von der Zahl der Wahlberechtigten verschieden. Daraus folgt, daß es unmöglich ist, den Erfolgswert der Stimmen im voraus gleichzumachen, demnach diese Ungleichheit als der gleichen Wahl widersprechend 
und das Wahlgesetz aus demselben Grund für verfassungswidrig zu erklären. Unsere Verfassung kennt kein Verfassungsgericht im eigentlichen Sinne, gibt also keinem Gericht die Möglichkeit, eine abstrakte Normenkontrolle über das Wahlgesetz auszuüben. Das Gericht soll die Verfassungsmäßigkeit der Gesetzesbestimmung regelmäßig in den Urteilsgründen beurteilen, soweit gesetzlich vorgesehen ist, daß eine Popularklage erhoben werden kann. Da diese Popularklage eine Streitigkeit bezüglich der Wirkung einer Wahl oder eines Gewähltwerdens ist, bedeutet die Nichtigerklärung einer Wahlgesetzbestimmung nichts anderes als die Vernichtung einer Wahl oder eines Gewähltwerdens, woraus folgt, daß die Revision der Verteilung der Mitgliederzahl im Wahlgesetz unmöglich ist, weil sonst das Parlament nicht zustande kommt.

\section{Versammlungsrecht}

In bezug auf die Gemeindesatzung ${ }^{3}$ über Versammlungen, Massenaufzüge oder Massenkundgebungen hat das Oberste Gericht durch Plenarentscheidung vom 20. Juli 1960 zwei Satzungen der Residenzstadt Tokio ${ }^{4}$ und der Stadt Hiroshima für verfassungsmäßig erklärt. In Hiroshima haben etwa 200 Personen, die an einer gerichtlichen Verhandlung über die Erklärung der Haftgründe nicht als Zuhörer teilnehmen konnten, ohne Erlaubnis des Sicherheitsausschusses an einem Aufzug auf der Straße vor dem Gericht teilgenommen. Als die Personen, die beschuldigt wurden, den Aufzug geleitet oder sich an ihm beteiligt zu haben, vor Gericht gestellt wurden, stellten sie die Verfassungsmäßigkeit der Satzung in Frage. Im zweiten Fall, als in Tokio etwa 3000 Studenten einen Aufzug durchführen wollten, hatte der Sicherheitsausschuß den Aufzug unter der Auflage erlaubt, daß eine bestimmte Art des Aufzuges (z. B. schlangen- oder wirbelförmig), die absichtlich gegen die Verkehrsordnung verstoße, verboten sei. Die Angeklagten leiteten den Aufzug und führten ihn wider die mit der Erlaubnis verbundene Auflage durch. Ebenso wie im ersten Fall machten sie im Prozeß die Verfassungswidrigkeit der Satzung geltend.

a) Das Oberste Gericht hatte in der Plenarentscheidung vom 24. November 1954 entschieden, daß eine Sicherheitssatzung mit Erlaubnisvorbehalt die Forderung der Verfassung nach größter Achtung der Menschenrechte verletzt, es sei denn,

\footnotetext{
3 Es gibt gegenwärtig 60 Satzungen, von denen 25 von den Provinzialvertretungen und 35 von den Gemeindevertretungen beschlossen sind. 53 Satzungen schreiben Polizeiverbote mit Erlaubnisvorbehalt betreffend Massenveranstaltungen vor, während 5 Satzungen bloß Anzeigepflicht anordnen. Ausnahme davon sind die Satzung der Präfektur Iwate, welche bezüglich Versammlungen Anzeigepflicht, bezüglich Massenaufzüge Erlaubnisvorbehalt vorschreiben, und diejenige der Stadt Kioto, welche bezüglich Versammlungen in geschlossenen Räumen Anzeigepflicht, bezüglich Versammlungen unter freien Himmel, Massenaufzügen Erlaubnisvorbehalt anordnen. Diese Satzungen regeln im allgemeinen Versammlungen an öffentlichen Orten, ausnahmsweise an öffentlichen Orten allein und Massenaufzüge an allen Orten. Erlaubnisanträge oder Anzeigen sollen innerhalb 72 oder 48 Stunden gestellt werden.

4 Die Sicherheitssatzung der Residenzstadt Tokio bestimmt wie folgt. $\int 1$ Versammlungen an der Straße oder sonstigen öffentlichen Orten und Massenkundgebungen an allen Orten bedürfen einer Erlaubnis des Sicherheitsausschusses der Residenzstadt Tokio.

\$ 2 Auf den Antrag soll der Sicherheitsausschuß Erlaubnis erteilen, es sei denn, daß die Durchführung der Massenveranstaltungen die öffentliche Ruhe unmittelbar und offenbar in Gefahr bringen würde. Er kann dennoch der Erlaubnis Bedingungen hinsichtlich folgender Artikel machen.

1. Vorbeugung einer Störung der Amtsgeschäfte.

2. Kontrolle eines Mitnehmens der Feuerwaffen, Mordwaffen oder sonstiger gefährlicher Gegenstände.

3. bis 6. (abgekürzt).

7. Änderung der Routen, Plätze oder Daten, wenn es zur Aufrechterhaltung öffentlicher Ordnung oder zur öffentlichen Gesundheitspflege unvermeidlich ist.
} 
daß die Satzung die erforderlichen Bestimmungen enthält, um einem Mißbrauch der vollziehenden Gewalt vorzubeugen. Vor allem sei es erforderlich zu bestimmen, erstens daß der Aufzug für erlaubt gelte, wenn bis zum Ablauf der gesetzlich festgesetzten Frist vor dem Termin die Erlaubnis nicht versagt werde, zweitens daß der Aufzug nur insoweit erlaubnisbedürftig sei, als er an bestimmten Orten oder auf bestimmte Art und Weise durchgeführt werde. Für diese Ansicht ist es gleichgültig, daß die Polizeierlaubnis nach pflichtmäßigem Ermessen des Sicherheitsausschusses getroffen werden soll. Aber die Gerichtsentscheidung wurde geändert. Nach dem Urteil des Obersten Gerichts vom 20. Juli 1960 verstoßen die oben genannten Satzungen mit dem allgemeinen Erlaubnisvorbehalt, nach dem jede Versammlung, soweit sie an öffentlichen Orten stattfinden, und jede Massenkundgebung an allen Orten der Erlaubnis bedürfen, nicht gegen Art. 21 der Verfassung, weil die Erlaubnis regelmäßig erteilt werden soll, es sei denn, daß der Ausschuß den Aufzug ganz klar als eine unmittelbare Gefahr für die öffentliche Ruhe ansieht. Nach der Ansicht des Gerichts liegt darin eine Bindung der Ermessensausübung, die es ermöglicht, den Ermessensmißbrauch im konkreten Fall nachträglich wirksam zu regulieren. Die Minoritätsansicht des späteren Urteils erklärte die Satzungen für verfassungswidrig, so wie die Majoritätsansicht des vorigen Urteils, es sei denn, daß man wegen der Verletzung ausdrücklicher Bestimmungen der Satzung die Aufhebung der auf Grund der Satzung getroffenen Verfügung ohne Schwierigkeit begründen kann. Nach dieser Ansicht sollen alle im Ermessen der Verwaltung stehenden Erlaubnisvorbehalte, vor allem wenn sie zur Beschränkung der Freiheit der Meinungsäußerung dienen, verfassungswidrig sein, während die Satzung mit Anzeigepflicht oder anderen Einrichtungen, die nur einen geringeren Ermessensspielraum gewähren, als verfassungsmäßig angesehen wird. Obgleich die Satzung mit Anzeigepflicht der betreffenden Behörde einen beträchtlichen Ermessensspielraum geben mag, weil der Sicherheitsausschuß daraufhin infolge der Prüfung der Einzelheiten der Anzeige den Aufzug im voraus verbieten kann, hat dennoch die oben erwähnte Plenarentscheidung vom 24. November 1954 die Satzung mit Erlaubnisvorbehalt mit derjenigen mit Anzeigepflicht identifiziert und nur insoweit für verfassungsmäßig erklärt. Durch Beschluß vom 9. Juni 1967 hat das Landgericht Tokio die einstweilige Aussetzung der Vollziehung auf eine Anfechtungsklage gegen die Nebenbestimmung der Erlaubnis verfügt. Zunächst hatte der Kläger einen Antrag um Erlaubnis des Aufzugs auf der Route A gestellt, demgegenüber hat die betreffende Behörde die Erlaubnis erteilt unter der Auflage, anstatt der Route A die Route B einzuschlagen. Der Kläger hat diese Auflage selbständig angefochten. Trotzdem zieht die Aufhebung dieser Auflage ipso iure die Nichtigkeit der Erlaubnis überhaupt nach sich, denn die Auflage ist der Erlaubnis wesentlich und die Erteilung der Erlaubnis in diesem Fall gilt als Versagung der beantragten Erlaubnis und Erteilung einer anderen. Meines Erachtens ist die Anfechtungsklage gegen die Auflage, den Aufzug auf der Route B durchzuführen, mangels Rechtsschutzbedürfnisses abzuweisen. Wenn man dagegen diese Anfechtungsklage durch die Verpflichtungsklage, die auf die Verurteilung zur Erteilung der Erlaubnis auf der Route A abzielt, ersetzen wollte, so würde die Verpflichtungsklage bei dem Justizgericht im Vergleich zum Verwaltungsgericht die autonome Entscheidung der Verwaltungsbehörde, anders ausgedrückt, die demokratische Herrschaft durch die parlamentarische Regierung und zugleich die Trennung der Verwaltung von der Justiz verletzen. Nun aber bezeichnet der Anfechtungsanspruch gegen die Versagung der Erlaubnis in bezug auf die Route A nichts 
anderes als den Anspruch auf die Wiederherstellung der noch nicht verweigerten Sachlage, die nicht schlechthin die Massenkundgebung rechtmäßig ermöglicht. Das Anfechtungsurteil kann zwar die Behörde binden, so daß die beantragte Erlaubnis erlassen werden muß, es sei denn, daß nachträglich eine Änderung der Sachlage oder der Rechtslage eintritt. In diesem Fall könnte man sogar die Aussetzung der Vollziehung mangels einer dringenden Notwendigkeit, schwer wiederherzustellende Schäden $\mathrm{zu}$ vermeiden, nicht verlangen, während bei der Anfechtungsklage auf Aufhebung des nach der Annahme der Anzeige erlassenen Verbots die Aussetzung der Wirkung des Verbots beantragt werden kann. Kurz, meiner Ansicht nach hat das Landgericht den Anfechtungsanspruch gegen die Versagung einer Erlaubnis mit demjenigen auf das bezüglich einer Anzeige erlassene Verbot verwechselt.

b) Die Sicherheitssatzung, die als Schrankenziehung der Freiheit der politischen Meinungsäußerung alle gegen das Gemeinwohl gerichteten Versammlungen regeln soll, ist verfassungswidrig, wenn sie dem Grundsatz der Verhältnismäßigkeit widerspricht. Demzufolge braucht man nicht zu bestrafen, wenn man auch den Aufzug ohne Erlaubnis infolge der Unterlassung des Erlaubnisantrags sowie den gegen die Nebenbestimmung oder das Gemeinwohl ausgeführten Aufzug mit Zuchthaus oder Geldstrafe bestrafen soll, weil ein solcher Aufzug an sich ohne Verletzung der materiellen Vorschriften durchgeführt wird und deshalb materiell nicht immer bestraft werden muß. Diese Strafbestimmung scheint zwar gegen den Grundsatz der Verhältnismäßigkeit zu verstoßen. Aber die Anklage steht im allgemeinen dem Ermessen des Staatsanwalts zu. Es besteht nicht immer ein zureichender Grund, die Strafbestimmung der Satzung deswegen als dem Grundsatz der Verhältnismäßigkeit widersprechend zu erklären, weil es nach der Satzung zu befürchten sein mag, irgendeinen wegen des Verstoßes gegen rein formelle Vorschriften gerichtlich zu verfolgen. Denn in diesem Fall würde die Angeklagte nicht verurteilt, sondern gegebenenfalls der betreffende Staatsanwalt wegen Ermessensmißbrauch seiner Amtsbefugnis bestraft werden.

Es scheint zwar aus demselben Grund verfassungswidrig zu sein, jede Massenkundgebung an allen Orten erlaubnisbedürftig zu machen. Aber es darf nicht immer mit Zuchthaus bestraft oder polizeilich gezwungen werden, wenn das Verfahren des Erlaubnisantrags oder der Anzeige nicht befolgt sind, es sei denn, daß die Massenbewegung materiell die öffentliche Sicherheit und Ordnung beeinträchtigt hat.

c) Die Auffassung, die Ermessensspielraum in sich schließende Satzung für verfassungswidrig zu halten, setzt ein starkes Mißtrauen gegen die Polizeibehörden voraus. Bei uns besteht die konservative Regierung seit dem Sturz des Kabinetts Katajama der Sozialistischen Partei im Jahre 1948. Daraus ergibt sich, daß eine parlaments- und regierungsfeindliche Haltung entstand. Solch eine Haltung setzt regelmäßig Vertrauen in die Ermessensentscheidung nicht einer Verwaltungsbehörde, sondern eines Gerichts wegen seiner politischen Neutralität. Der Beschluß des Ersten Senats des Obersten Gerichts vom 21. September 1960 hat den Beschluß des Landgerichts Tokio, der eine Verhaftung sui generis, die formell von der eigentlichen Strafe unterschieden wird, ausgesprochen hat, für verfassungsmäßig erklärt. In einem Verfahren, in dem es darum ging, daß dem Verhafteten der Grund der Verhaftung sofort in öffentlicher Gerichtssitzung in Gegenwart seines Rechtsanwalts angegeben werden sollte, stellte ein Rechtsanwalt ein Ablehnungsgesuch 
und begründete es damit, daß der betreffende Richter voreingenommen sei und keine Urteilsfähigkeit darüber habe, ob die Tatbestandsmerkmale der Haft erfüllt seien oder nicht. Der Richter forderte, diese Aُußerung zu widerrufen und ihm Abbitte zu leisten. Als der Rechtsanwalt diese Forderung ablehnte, nahm der Richter den Rechtsanwalt wegen schwerer Verletzung des gerichtlichen Ansehens in Haft, verurteilte ihn nach der nicht öffentlichen Untersuchung zu zwanzig Tagen Haft und wies den Antrag auf Beweisaufnahme wegen unmittelbarer Kenntnis des Richters ab. Dagegen bestand der Verhaftete auf der Verfassungswidrigkeit der Haft, weil die mit dem Strafprozeß zusammenhängenden verfassungsrechtlichen Garantien auf diese Haft sui generis, die materiell eine Art Strafe sei, anzuwenden seien, und begründete seinen Einwand damit, daß er ohne Haftbrief verhaftet worden sei, daß auf sein Verlangen der Grund der Verhaftung nicht sofort in der öffentlichen Gerichtssitzung angegeben worden sei, daß er ohne Anklage eines Staatsanwalts gerichtlich verfolgt worden sei und daß ihm das Recht, einen Rechtsanwalt für sich heranzuziehen, nicht gewährleistet worden sei. Das Oberste Gericht hat diese Einrede abgewiesen, weil die oben erwähnte Haft weder eine Kriminal- noch eine Verwaltungsstrafe sei, sondern sich zwangsläufig aus der Aufgabe der Justizgewalt und ihrer ordnungsmäßigen Führung ergebe, mit anderen Worten eine um der Selbsterhaltung und der Notwehr der Justiz willen der Justizgewalt immanente Befugnis sei. Anders als das anglo-amerikanische Rechtsinstitut der Beleidigung des Gerichts (contempt of court), das als Vergehen im eigentlichen Sinne anerkannt ist, braucht unser System, das keine Strafe nach sich zieht, kein besonderes Verfahren, weil der Richter persönlich das rechtswidrige Handeln gesehen und gehört hat, also zugleich als Anzeigender, Geschworener und Vollstrecker das Ansehen einer Gerichtssitzung mittels sofortiger Entscheidung wiederherstellen darf. Die Vor- und Nachteile der Lösung durch richterliche Gewalt halten in dieser Hinsicht das Gleichgewicht und für welche man sich entscheiden soll, ist schwer zu sagen. Die richterliche Gewalt ist leicht zu mißbrauchen. Ihrem Schutz durch sich selbst liegt die Behutsamkeit und die Entschlossenheit, also die sich selbst widersprechenden Tugenden eines Richters zugrunde. Der oben erwähnte Fall betrifft die Bestrafung von Gewalttaten der Linken, während hinsichtlich der Unruhe an den Hochschulen viele Strafsachen gegen linke Studenten eingeleitet wurden, in denen man in den Gerichtssitzungen oft das Verfahren zu verzögern versuchte, sich der Prozeßführung eines Richters nicht unterwarf und endlich in Abwesenheit verurteilt wurde. Die radikalen Juristen fürchten, sie müssen sich auf Mißbrauch von seiten des Richters gefaßt machen. Sie verfechten die Einschränkung der Anwendung des Gesetzes betreffend die Aufrechterhaltung der Ordnung in den Gerichtssitzungen. Derartige Rechtsanwälte, Staatsanwälte und Richter gehören im allgemeinen zum "Jugendverein der Juristen“. Neuerdings hat das Justizministerium als Beteiligter am Prozeß einen dieser Richter abgelehnt, und bald danach hat der Chefrichter des Obersten Gerichts Ishida am 2. Mai 1970 die Mahnung ausgesprochen, daß die Freiheit der politischen Meinungsäußerung eines Richters als Staatsbürger nicht nur innerhalb, sondern auch außerhalb seiner Amtsführung eingeschränkt sei, um die Rechte mehrerer durch ein Urteil betroffener Beteiligten des Streitverfahrens zu gewährleisten, und er hat davor gewarnt, die Unparteilichkeit der Entscheidungen in Frage $\mathrm{zu}$ stellen, indem ein Richter irgendeinen politischen Verein organisiert oder ihm beitritt. Denn im Vergleich mit der politischen Neutralität eines gewöhnlichen Beamten wird dem Richter die besondere höhere Unparteilichkeit bei der Ausübung seiner Amtsgewalt verfassungsrechtlich gewährleistet, desto größer ist 
seine Verantwortung, pflichtgemäß gerecht zu urteilen. Freilich teilen viele Juristen seine Meinung, aber einige verfechten die Ansicht, daß der Richter in seiner Meinungs- und Gewissensfreiheit nicht verletzt werden darf und sogar der Eintritt in irgendeinen politischen Verein den betreffenden Richter nicht untauglich macht, soweit die Entscheidungen des Vereins ihn nicht binden. Der Antrag auf die Anklage gegen Richter Ishida, um ein Verfahren auf Amtsentfernung vor dem besonderen Gerichtshof, gebildet aus den Mitgliedern beider Häuser (Art. 64 Verf.), $\mathrm{zu}$ eröffnen, hat der Anklageausschuß zurückgewiesen. Kurz, sowohl das Vertrauen auf den Richter als auch das Mißtrauen gegen die Verwaltungsbehörde beruht bei uns nicht auf der schlichten Rechtslogik, sondern auf der Sachlage, ob wirklich von den Richtern regierungsfeindliche, revolutionäre Haltung zu erwarten ist und ob ein Regierungswechsel in nächster Zeit möglich sein mag.

\section{Streik im öffentlichen Dienst}

Der Parole des Arbeitskampfs im Frühjahr folgend, haben die Angeklagten eines Strafprozesses viele Beamten und Angestellten am Zentralpostamt Tokio dazu überredet, an der in den Dienststunden stattfindenden Dienststellenmassenversammlung vom 20. März 1966 teilzunehmen. 38 Personen haben eingewilligt und ihre Dienststelle zwei bis sechs Stunden verlassen, ohne ihre Dienstobliegenheiten zu erfüllen. Nach der Anklageschrift des Staatsanwalts haben sich die Angeklagten der Anstiftung zu dem Verbrechen schuldig gemacht, das jemanden, der mit Postgeschäften beschäftigt ist und vorsätzlich die Handhabung der Postsachen unterläßt oder verzögert, mit bis zu einem Jahr Zuchthausstrafe oder mit einer Geldstrafe von bis $\mathrm{zu} 20,000$ Yen bedroht. Das Langericht Tokio hat die Angeklagten wegen rechtmäßiger Arbeitseinstellung gemäß $\int 1$ Abs. 2 Gewerkschaftsgesetz freigesprochen. Trotzdem gilt die Nichtbehandlung der Postsachen durch Abwesenheit von der Arbeitsstelle als die rechtmäßige Geschäftsführung störende Arbeitseinstellung. Jedoch enthält das Gesetz betreffend die Arbeitsverhältnisse der Körperschaften des öffentlichen Unternehmens ${ }^{5}$, das sich vor allem auf Post und Eisenbahn bezieht, keine Strafbestimmungen, sondern bestimmt sogar ausdrücklich die Ausschließung der Anwendung des $₫ 8$ Gewerkschaftsgesetz in bezug auf die Befreiung von der zivilrechtlichen Verantwortung, während es von der Ausschließung der Anwendung des $\ 1$ Abs. 2 Gewerkschaftsgesetz in bezug auf die Befreiung von der strafrechtlichen Verantwortung schweigt. Wenn man bedenkt, daß der Dienst der Postbeamten und derjenige der gewöhnlichen Arbeiter im großen und ganzen als gleichartig anzusehen ist, und daß der Streik der gewöhnlichen Beamten zwar verboten, die Strafbestimmung wegen Verletzung des Streikverbotes dennoch speziell auf diejenigen, die sich zum Streik verschwören, dazu aufreizen oder aufhetzen, anzuwenden ist, so besteht der Zweck des Gesetzes betreffend die erwähnten Arbeitsverhältnisse offenbar darin, den Streik als solchen nicht zu bestrafen. Das obere Gericht Tokio hat die Berufung von seiten der Angeklagten verworfen, und diese Entscheidung damit begründet, daß es über die Unrecht-

5 Das Gesetz betreffend die Arbeitsverhältnisse der Körperschaften des öffentlichen Unternehmens, 1949, bezieht sich auf die Staatseisenbahn, das Tabakmonopol und die Telegraphen- und Telefonkorporation, mit anderen Worten drei öffentliche Anstalten mit juristischer Persönlichkeit. Außerdem soll es seit 1952 auf den mit dem Postwesen (320000 Personen eingeschlossen), dem Forstamt (39000 Personen), der Münzen (1 000), der Staatsdruckerei (7 400) und dem Alkoholmonopol (1 100) sich beschäftigenden Personen, anders ausgedrückt, fünf Unternehmungsabteilungen der Staatsorganisation angewendet werden. 
mäßigkeit des Streiks keiner weiteren Ausführung bedarf, weil der Streik dem Personal des Postamts verboten und damit also auch das Streikrecht entzogen ist, so daß $₫ 1$ Abs. 2 Gewerkschaftsgesetz demnach hier keine Anwendung finden kann.

Nach den Leitsätzen der Plenarentscheidung des Obersten Gerichts vom 26. Oktober 1966 finden Art. 25, 27 und 28 der Verfassung auf die Beamten und das Personal der Körperschaften des öffentlichen Unternehmens Anwendung. Wenn man aus dem Wesen des Beamten als Diener der Gesamtheit (Art. 15 Abs. 2 der Verfassung) seine Arbeitsgrundrechte einschränken will, so setzt das Harmonie und Gleichgewicht zwischen Art. 15 der Verfassung einerseits, und der Garantie der Vermögensrechte (Art. 29) und der Arbeitsgrundrechte (Art. 28) andererseits voraus. Daraus ergibt sich, daß auch im Falle der Angestellten und anderer Personen die Einschränkung des Streiks, welche die Interessen des ganzen Lebens der Bevölkerung gewährleisten und den Grundrechten immanent sind, sich auf das notwendige Minimum beschränken muß und sogar gleichzeitig Ersatzmaßnahmen vorsehen muß. Kurz, $\ 17$ Abs. 1 Gesetz betreffend die erwähnten Arbeitsverhältnisse verstößt nicht gegen Art. 28 und 18 der Verfassung. Das Streikverbot fordert Vermittlung, Schlichtung, Schiedsurteil usw. mittels eines Verwaltungsausschusses als Ersatzmaßnahme. Die Verletzung des Streikverbots soll nichts anderes als disziplinar- oder zivilrechtliche Verantwortung wie z. B. Entlassung, Schadensersatz nach sich ziehen. Die Geschichte des Rechtsinstituts zeigt, daß die Sanktion beim Gesetz betreffend die erwähnten Arbeitsverhältnisse milder sein soll als die beim Beamtengesetz. Schließlich ist die Anwendung des $\ 1$ Abs. 2 Gewerkschaftsgesetz nicht immer ausgeschlossen. Anders ausgedrückt, eine Strafe soll nicht verhängt werden, es sei denn, daß der Streik politischen Zwecken dient, Gewalttaten mit sich bringt oder erfahrungsmäßig übermäßig langwierige, für das Leben des Volkes schwere Störungen hervorruft. Die Minoritätsansicht der Richter Okuno, Kusaka und Ishida lautet: $\mathrm{Da}$ über die Rechtswidrigkeit einer Handlung auf allen Rechtsgebieten einheitlich entschieden werden muß, ist es widerspruchsvoll, denselben Streik auf dem Gebiet des Gesetzes betreffend die genannten Arbeitsverhältnisse für rechtswidrig und zugleich auf dem Gebiet des Strafgesetzbuchs für rechtmäßig zu halten. Daher können weder die Beamten noch die Angestellten der Körperschaften des öffentlichen Unternehmens mangels Streikrechts rechtmäßig Streik anfangen. Vorausgesetzt doch, daß auf die betreffenden Personen $\ 1$ Abs. 2 Gewerkschaftsgesetz Anwendung findet, gäbe es keinen wesentlichen Unterschied der Verantwortung im Vergleich mit gewöhnlichen Arbeitern. Daß das Gesetz betreffend die erwähnten Arbeitsverhältnisse die Anwendung des $\ 1$ Abs. 2 Gewerkschaftsgesetz nicht ausschließt, hat den Grund darin, daß man diese Bestimmung, den Streik ausgenommen, auf kollektive Verhandlungen anwenden wollte. Da die Rechtswidrigkeit einer Handlung im Zusammenhang mit dem beeinträchtigten Rechtsgut relativ entschieden werden kann - z. B. die chirurgische Operation eines Nichtarztes auf dem Gebiet des Arztgesetzes ist als rechtswidrig, in bezug auf Verletzungsverbrechen dagegen als rechtmäßig anzusehen -, ist die Zuwiderhandlung gegen das Streikverbot, das auf den Schutz des Gemeinwohls zielt, nicht immer als Geschäftsstörung mit Gewalt anzusehen, die den Schutz des Unternehmers zum Zweck hat. Trotzdem hat das Vergehen des $\ 79$ Postgesetz ebenso wie das Streikverbot ( $\mathbb{1} 17$ Gesetz betreffend die erwähnten Arbeitsverhältnisse) das Gemeinwohl zum gemeinsamen Rechtsgut. Daraus folgt, daß man bloß um der Relativität der Rechtswidrigkeit willen die Minoritätsansicht nicht widerlegen kann. Des- 
halb bezeichnet die Majoritätsansicht nichts anderes als die Auffassung, daß der Streik noch nicht die strafbare Rechtswidrigkeit erreicht.

\section{Recht auf Fürsorge}

Der Kläger $\mathrm{X}$ war seit langem wegen Lungentuberkulose in einem staatlichen Sanatorium. Er erhielt sowohl eine monatliche Unterstützung von 600 Yen für den täglichen Bedarf als auch die Unterstützung mit Nahrungsmitteln im Sanatorium. Seit August 1956 sandte ihm sein älterer Bruder monatlich 1500 Yen. Deshalb stellte die zuständige Behörde nach dem Subsidiaritätsprinzip, das für die Unterstützung gilt, die oben erwähnte Versorgung ein und erlegte dem $\mathrm{X}$ als Teil der Unterstützung im Sanatorium sogar die Restsumme von 900 Yen auf. Weil X trotz der Unterstützung durch seinen Bruder keinen Vorteil für sich hatte, erhob er bei dem zuständigen Präfekten Einspruch gegen den die Unterstützung ändernden Verwaltungsakt und führte beim Wohlfahrtsminister Beschwerde darüber. Nachdem der Einspruch und die Beschwerde zurückgewiesen worden waren, erhob $\mathrm{X}$ gegen den Wohlfahrtsminister eine Anfechtungsklage, in Wahrheit Klage auf Feststellung der Nichtigkeit. Dabei machte X geltend, daß die Summe von 600 Yen für den täglichen Bedarf nach der Lebensunterstützungsnorm so unzulänglich sei, daß er ergänzende Mahlzeiten einnehmen müsse, um voll ernährt zu sein. Seiner Ansicht nach betrugen seine geringsten Lebenskosten monatlich 1000 Yen.

Der Kern dieser Klage lag in der Entscheidung, ob die Geldsumme von 600 Yen für den täglichen Bedarf hoch genug oder zu niedrig sei und ob die Behörden den Betrag, der sich aus dem Unterschied zwischen der nach der Meinung des Klägers richtigen Unterstützungsnorm und der geltenden Summe von 600 Yen ergibt, zurückfordern könne. Wenn dem Antrag des Klägers stattgegeben würde, würde das die Lösung eines allen Kranken in Sanatorien des ganzen Landes gemeinsamen Problems beeinflussen. Vor allem wirkt sich die Entscheidung über die geringsten Lebenskosten auf die ganz verschiedenartigen Fürsorgeleistungen und den Minimallohn aus. In diesem Prozeß kam es darauf an (1), ob Fürsorgebedürftige oder Fürsorgeempfänger eine Unterstützungsverfügung wegen ihrer Rechtswidrigkeit anfechten können (2), ob die Lebensunterstützungsnorm des Wohlfahrtsministeriums wirklich gerichtlich nachprüfbar ist (3), was als das Mindestmaß gesunden und kultivierten Lebens (Art. 25 der Verfassung) substantiell bezeichnet werden soll. Die Fragen zu 1 und 2 haben die 1. und 2. Instanz positiv beantwortet, dagegen gehen die Auffassungen beider Instanzen darüber auseinander, ob ein Gericht das Ermessen des Wohlfahrtsministeriums anerkennen muß. Die 1. Instanz hat die abweisende Verfügung des Wohlfahrtsministeriums aufgehoben, während die Berufungsinstanz die Klage deshalb als unbegründet abgewiesen hat, weil es keine Frage der Rechtmäßigkeit, sondern der Zweckmäßigkeit sei, wenn das Gericht die Norm des täglichen Bedarfs für zu niedrig hält. $X$ ernannte einen Prozeßvertreter und legte Revision ein. Er adoptierte ein Ehepaar Y und Z, das er mit der Fortführung des Prozesses beauftragte. Nach seinem Tode haben $Y$ und $Z$ bei dem Obersten Gericht die Übernahme des Streitverfahrens beantragt. Nach $\int 213$ ZPO wird das Verfahren trotz des Todes des Prozeßbeteiligten während eines rechtshängigen Verfahrens nicht unterbrochen, wenn ein Prozeßvertreter ernannt ist. Anders ausgedrückt: ein besonderer Antrag zur Übernahme des Verfahrens ist nicht erforderlich, sondern es ist im Gegenteil unvermeidlich, daß 
der Erbe auch eine Stellung des Beteiligten im Prozeß übernimmt. Dies hatte das Gericht als Vorfrage des Sachurteils von Amts wegen zu untersuchen. Bei vermögensrechtlichen Prozessen taucht die Frage nach der Prozeßübernahme im allgemeinen nicht auf, weil der Erbe die Stellung der Beteiligten des Prozesses übernimmt, indem er den Streitgegenstand, nämlich das Vermögensrecht des Erblassers, erbt. Nach dem Urteil des Obersten Gerichts in der Plenarentscheidung vom 24. Mai 1967 lautet die Majoritätsansicht folgendermaßen: Der Fürsorgeanspruch des Klägers ist weder übertragbar noch vererblich, weil er nach dem Unterstützungsgesetz höchstpersönlich ist. Daher erlischt sowohl dieser Anspruch an sich als auch der Anspruch auf Leistung des während seines Lebens entstandenen, im Verzug befindlichen Versorgungsgeldes, wenn der Berechtigte stirbt. Die oben erörterte Rückforderung aus ungerechtfertigter Bereicherung setzt den Fürsorgeanspruch voraus, ist also genauso wie dieser, nicht vererblich. Daraus ergibt sich, daß der Prozeß mit dem Tode des Klägers zu Ende war.

Dagegen lautet die Minoritätsansicht: Wenn die oben erwähnte Entscheidung über die Unterstützung rechtswidrig ist, braucht der Kläger dem Staat nicht seine medizinischen Ausgaben zu erstatten. Daraus folgt, daß die Rückforderung des Klägers in diesem Falle zum Unterschied von dem Versorgungsanspruch vererblich ist. Um in Zukunft den Anspruch des Erblassers geltend zu machen, ist es erforderlich, daß der Erbe die Anfechtung der betreffenden Entscheidung verlangt. Darum muß man die Prozeßübernahme durch den Erben anerkennen. Um einen Anspruch, der keinen gegenwärtigen Streitgegenstand bildet, künftig geltend zu machen, darf man nach der Zivilprozeßordnung einen Prozeß nicht fortführen, während nach $\int 9$ der Verwaltungsprozeßordnung jedem, der an der Anfechtung einer Verfügung oder einer Entscheidung ein rechtliches Interesse hat, die Befugnis zur Anfechtungsklage zuerkannt wird, selbst wenn die Wirkung einer Verfügung usw. wegen Ablauf der Klagefrist usw. erloschen ist.

Der Unterstützungsanspruch wird zwar vom Unterstützungsgesetz gewährleistet, um den Gedanken der Garantie der Daseinsberechtigung zu verwirklichen, aber es handelt sich darum, ob die Verfassung unmittelbar objektiv die Lebensnorm, verbunden mit den geringsten Lebenskosten, festsetzen und aufrechterhalten kann oder ob die Verfassungsgarantie im Gegenteil sich nur so weit erstrecken kann, wie es die Regierung für notwendig hält, um die geringsten Lebenskosten zu bestreiten. Nach der ersten Meinung ist der Versorgungsanspruch ein unmittelbar mit der Daseinsberechtigung verbundenes aktuelles Recht. Der Normsetzungsakt des Wohlfahrtsministers ist ein Rechtsvollziehungsakt, der den objektiven Inhalt der Norm rationell ergründet und pekuniär veranschlagt. Demgemäß ist ein Beurteilungsfehler des Normsetzungsakts verfassungswidrig und gerichtlich nachprüfbar. Nach der zweiten Meinung ist der Normsetzungsakt des Wohlfahrtsministers, so wie die Bekanntmachung des offiziellen Preises, zwar eine allgemeine Verfügung im formellen Sinne, zugleich aber ein rechtsergänzender Rechtsetzungsakt im materiellen Sinne. Darum ist die Beurteilung in bezug auf das Mindestmaß gesunden und kultivierten Lebens (Art. 25 der Verfassung) dem politischen Ermessen des Wohlfahrtsministers überlassen. Ein solcher Ermessensfehler ist eine bloße Frage der Unzweckmäßigkeit, die nur dann gerichtlich nachprüfbar ist, wenn die Entscheidung dem Sinne der Verfassungsbestimmung und dem Unterstützungsgesetz widerspricht, z. B. entgegen der Lebenswirklichkeit eine äußerst geringe Norm setzt, was als Ermessensüberschreitung oder Ermessensmißbrauch angesehen werden muß. 
Der Mehrheitsansicht und der des Richters Tanaka zufolge soll der Begriff des geringsten und dennoch gesunden, kultivierten Lebens immer der jeweiligen Sachlage nach entsprechend bestimmt werden, während niemand den konkreten Begriffsinhalt mit rechnerischer Genauigkeit erfassen könne. Es sei eine Fiktion, diesen Betrag in einem bestimmten Zeitpunkt für objektiv definierbar zu halten. $\mathrm{Da}$ die Unterstützung, abgesehen von einer allgemeinen Norm, für den einzelnen Unterstützungsbedürftigen persönlich nach der Sachlage seiner wirtschaftlichen Verhältnisse entschieden werden soll, widerspricht dem Sinne des Gesetzes, daß diese Entscheidung einheitlich getroffen werden muß. Richter Okuno ist der Meinung: Das Recht auf Dasein in Art. 25 der Verfassung werde nicht als Freiheitsrecht, sondern als positiver Anspruch gewährleistet. Deshalb teilt er die letztere Meinung. Wenn es nachher vorkomme, daß einzelne Artikel der Unterstützungsnorm dem Grundgedanken der Norm widersprächen, so könne man trotzdem die betreffenden Artikel nicht schlechthin für verfassungswidrig halten, denn der Zweck der Norm bestehe darin, das Ermessen der zuständigen Behörden auszuschließen und die Unterstützung gerecht und unparteiisch durchzuführen. Wenn das Wesen der Unterstützung auch durch die Veränderung der bisherigen Umstände den Lebensverhältnissen nicht genau entsprechen möge, so werde doch ein solches Auseinanderfallen für unvermeidlich gehalten und damit rechtlich zugelassen, jedenfalls sofern es innerhalb einer zur Normsetzung nötigen Frist entstanden ist und die Abweichung der Norm von der Lebenswirklichkeit in dem bestimmten Zeitpunkt nicht so groß ist, daß der Sinn und Zweck der Verfassung und des Unterstützungsgesetzes verletzt werden. Deshalb gibt es keinen beträchtlichen Unterschied zwischen beiden Ansichten. 